\title{
Experimental realization of a local-to-global noise transition in a two-qubit optical simulator
}

\author{
Claudia Benedetti $\odot,{ }^{1}$ Valeria Vento $\odot,{ }^{1}$ Stefano Olivares $\odot,{ }^{1,2}$ Matteo G. A. Paris,${ }^{1,2}$ and Simone Cialdi ${ }^{1,2}$ \\ ${ }^{1}$ Dipartimento di Fisica “Aldo Pontremoli," Università degli Studi di Milano, I-20133 Milano, Italy \\ ${ }^{2}$ INFN, Sezione di Milano, I-20133 Milano, Italy
}

(Received 26 November 2019; accepted 4 March 2020; published 30 March 2020)

\begin{abstract}
We demonstrate the transition from local to global noise in a two-qubit all-optical quantum simulator subject to classical random fluctuations. Qubits are encoded in the polarization degree of freedom of two entangled photons generated by parametric down-conversion (PDC) while the environment is implemented by using their spatial degrees of freedom. The ability to manipulate with high accuracy the number of correlated pixels of a spatial-light-modulator and the PDC spectral width allows us to control the transition from a scenario where the qubits are embedded in local environments to the situation where they are subject to the same global noise. We witness the transition by monitoring the decoherence of the two-qubit state.
\end{abstract}

DOI: 10.1103/PhysRevA.101.032348

\section{INTRODUCTION}

Quantum simulators are controllable quantum systems, usually made of qubits, able to mimic the dynamics of other, less controllable, quantum systems [1,2]. Quantum simulators make it possible to design and control the dynamics of complex systems with a large number of degrees of freedom, or with stochastic components [3-7]. In turn, open quantum systems represent a fundamental test-bed to assess the reliability and the power of a quantum simulator. The external environment may be described either as a quantum bath, or a classical random field which, in general, lead to different system evolutions. However, in the case of pure dephasing, the effects of a quantum bath are equivalent to those provoked by random fluctuations [8]. For this reason, together with the fact that it is a ubiquitous source of decoherence that jeopardizes quantum features, dephasing noise plays a prominent role in the study of open quantum systems.

Pioneering works on the controlled simulation of singlequbit dephasing channels appeared a few years ago $[9,10]$, whereas the realization of multiqubit simulators is still missing. In fact, the simulation of multiqubit systems is not a mere extension of the single-qubit case since composite systems present features that are absent in the single-component case, e.g. entanglement [11-15]. Moreover, multipartite systems allow us to analyze the effects of a global source of noise against those due to local environments. Understanding the properties of the local-to-global (LtG) noise transition is in turn a key task in quantum information, both for quantum and classical environments, since it sheds light on the mechanisms governing the interaction between the quantum system and its environment, providing tools to control decoherence [16-21].

We present here an all-optical implementation of the whole class of two-qubit dephasing channels arising from the interaction with a classically fluctuating environment. The qubits are encoded in the polarization degree of freedom of a photonpair generated by parametric down-conversion (PDC), while the spatial degrees of freedom are used to implement the environment. Different realizations of the noise are randomly generated and imprinted on the qubits through a spatial-light modulator (SLM). The ensemble average is then performed by collecting the photons with a multimode fiber. With our simulator there is no need to work at cryogenic temperatures and we are able to simulate any conceivable form of the environmental noise, independently of its spectrum.

In particular, here we exploit our simulator to demonstrate the transition from a local-environment scenario, where each qubit is subject to an independent source of noise, to a global environment where both qubits feel the same synchronous random fluctuations. There are two different mechanisms that may lead to this transition. The first one appears when two local environments become correlated due to the action of some external agent, and one moves from local to global noise as the two environments become fully correlated. In the second scenario, the two qubits are placed in the same environment, but at a distance that is much larger than the correlation length of the noise. As the distance between the qubits is reduced, they start to feel similar environments, until they are within the correlation length of the environment and thus subject to the same common source of noise. The two situations are illustrated in Fig. 1. In the first case, two initially different environments (cottages) become gradually identical as far as correlations are established (by putting bricks), whereas in the second case the two qubits (persons) are initially far apart, but they end up feeling the same environment (cottage) as long as their distance is reduced.

The dephasing map of two noninteracting qubits arising from a classical environment is generated by the dimensionless Hamiltonian:

$$
H(t)=X_{1}(t) \sigma_{z}^{(1)} \otimes \mathbb{I}^{(2)}+X_{2}(t) \mathbb{I}^{(1)} \otimes \sigma_{z}^{(2)},
$$

where $\sigma_{z}$ is the Pauli matrix, $\mathbb{I}$ the identity matrix, $X(t)$ is a stochastic process and the labels 1 and 2 denote the two qubits. Since our aim is to give a proof of principle of the $\mathrm{LtG}$ transition and we are not interested at this stage in the specific form of the noise, we fix the stochastic process to be a random telegraph noise (RTN). It follows that $X_{k}(t)= \pm 1$ is a dichotomous variable which jumps between two values 

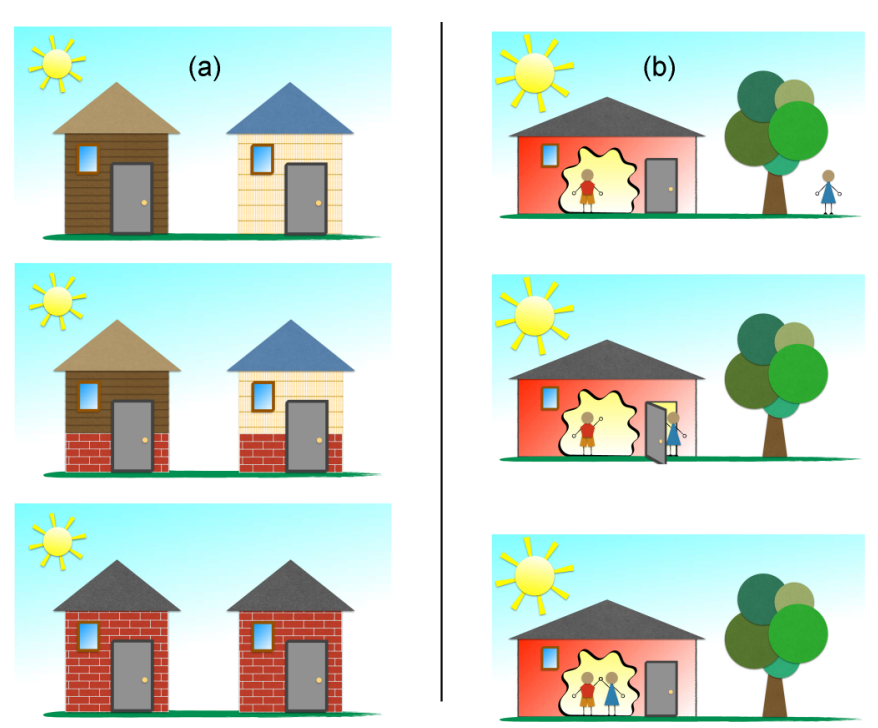

FIG. 1. Cartoon description of the two scenarios leading to the LtG transition. On the left: LtG transition after establishing correlations between the two environments. On the right: LtG transition as the qubits approach each other.

with a certain switching rate $\gamma$ that determines the correlation length of the noise through the autocorrelation function $C(t)=\langle X(t) X(0)\rangle=e^{-2 \gamma t}$. The symbol $\langle\ldots\rangle$ denotes the ensemble average over all possible realizations of the RTN. In Eq. (1) it is possible to identify two complementary regimes: If $X_{1}(t)$ and $X_{2}(t)$ are two identical but independent processes, then we are in the presence of local environments and each qubit is subject to its own noise. On the other hand, if $X_{1}(t)=$ $X_{2}(t)$, at all times, then the fluctuations are synchronized (perfectly correlated) and the qubits interact with the same global environment. The generated dynamics in these two scenarios are very different and this can be witnessed, for example, by looking at the behavior of entanglement. What happens in between these regimes is unexplored territory.

\section{THEORETICAL MODEL}

To address the local-to-global noise transition, we first need to compute the two qubits dynamics in the presence of classical noise. Starting from an initial Bell state

$$
\left|\psi_{0}\right\rangle=\frac{1}{\sqrt{2}}(|\mathrm{HH}\rangle+|\mathrm{VV}\rangle)
$$

the system density matrix is obtained as $\rho(t)=$ $\left\langle U(t) \rho_{0} U^{\dagger}(t)\right\rangle$, where $\rho_{0}=\left|\psi_{0}\right\rangle\left\langle\psi_{0}\right|$, the evolution operator has the form:

$$
U(t)=\exp \left[-i \int H(s) d s\right]=\exp [-i \varphi(t)],
$$

where $\varphi(t)=\int H(s) d s$ is the noise phase. The elements of $\rho(t)$ in the polarization basis $\{|\mathrm{HH}\rangle,|\mathrm{HV}\rangle,|\mathrm{VH}\rangle,|\mathrm{VV}\rangle\}$ are $\rho_{11}(t)=\rho_{44}(t)=\frac{1}{2}$ and $\rho_{14}(t)=\rho_{41}^{*}(t)=\frac{1}{2} \Gamma(t)$, and all other elements are zero. The coherence factor $\Gamma(t)$ depends on the nature and the correlations of the noise. In particular, it was shown [13] that, for local environments (LE) and global environments (GE), the coherence factor takes the forms

$$
\Gamma_{\mathrm{LE}}(t)=\left\langle e^{2 i \varphi(t)}\right\rangle^{2}, \quad \Gamma_{\mathrm{GE}}(t)=\left\langle e^{4 i \varphi(t)}\right\rangle,
$$

where the averages of the exponential moments are given by $\left\langle e^{m i \varphi(t)}\right\rangle=e^{-\gamma t}\left(\cosh \delta_{m} t+\gamma / \delta_{m} \sinh \delta_{m} t\right) \quad$ with $\delta_{m}=\left(\gamma^{2}-m^{2}\right)^{1 / 2}$. The entanglement $\mathcal{E}$ between the qubits is given by $\mathcal{E}(t)=|\Gamma(t)|$ in both LE and GE cases.

The realization of the qubit state $\rho(t)$ requires the simultaneous generation of a large number of stochastic trajectories of the noise. Our experimental apparatus allows us to obtain the average over the realizations in parallel, exploiting the spatial and the spectral degrees of freedom of the photons. In particular, in our experimental setup the following state is generated:

$$
\begin{aligned}
\left|\psi_{\mathrm{SE}}(t)\right\rangle= & \frac{1}{\sqrt{2}} \int d x_{1} d x_{2} f\left(x_{1}, x_{2}\right) \\
& \times\left[\left|\mathrm{H} x_{1} \mathrm{H} x_{2}\right\rangle+e^{i\left[\varphi_{1}\left(x_{1}, t\right)+\varphi_{2}\left(x_{2}, t\right)\right]}\left|\mathrm{V} x_{1} \mathrm{~V} x_{2}\right\rangle\right],
\end{aligned}
$$

where $f\left(x_{1}, x_{2}\right)$ is the spatial correlation function between the two photons, the $\varphi_{k}\left(x_{k}, t\right)$ are the noise phases, and $\left|\mathrm{P}_{1} x_{1} \mathrm{P}_{2} x_{2}\right\rangle$ denotes a state where the photon 1 (2) has polarization $\mathrm{P}_{1}$ $\left(\mathrm{P}_{2}\right)$ and is in position $x_{1}\left(x_{2}\right)$. In this scenario, the stochastic trajectories are encoded in the spatial degree of freedom and the state $\rho(t)$ is obtained by tracing out $x_{1}$ and $x_{2}$. Finally, as we show below, we employ the spectral degree of freedom to define the degree of spatial correlation between the two photons.

\section{EXPERIMENTAL REALIZATION}

Our experimental setup is schematically depicted in Fig. 2. The pump is generated from a $405 \mathrm{~nm} \mathrm{cw} \mathrm{InGaN} \mathrm{laser}$ diode. The laser beam passes through an amplitude modulator, composed of a half-wave plate and a polarizing beam-splitter (PBS), and then through another half-wave plate to set the polarization. Subsequently, the polarized $405 \mathrm{~nm}$ beam goes through a telescopic system composed of two lenses $L_{t 0}$ and $L_{t 1}$ with respective focal lengths $f_{t 0}=100 \mathrm{~mm}$ and $f_{t 1}=$ $75 \mathrm{~mm}$, with the double purpose of collimating the beam and optimizing its size in order to maximize the detection efficiency. After the telescopic system there are three 1-mm-long crystals that compensate the delay time introduced by the PDC crystals. To generate the entangled state in the polarization we use two 1-mm-long crystals of beta barium borate (BBO) [22]. On each branch of the PDC, a lens $L$ with focal length $f=200 \mathrm{~mm}$ at $810 \mathrm{~nm}$ is placed a distance $f$ from the BBO. On the Fourier plane (a distance $f$ from the lens $L$ ) there is the SLM, which is a one-dimensional liquid-crystal mask with 640 pixels of width $100 \mu \mathrm{m} /$ pixel. Each pixel imprints a computer-generated phase on the horizontal-polarized component. Photons then pass through polarizers and are finally focused into a multimode fiber through the couplers $C_{1}$ and $C_{2}$ and into a photon-counting module. The signal beam, before the detection, passes through a spectral selector, which consists of two gratings and two lenses building an optical $4 f$ system. In the Fourier plane of this apparatus, we use a mechanical slit to select the desired spectral width [23].

Since each pixel of the SLM has a finite width, we may substitute the integral with a sum over the pixel positions 


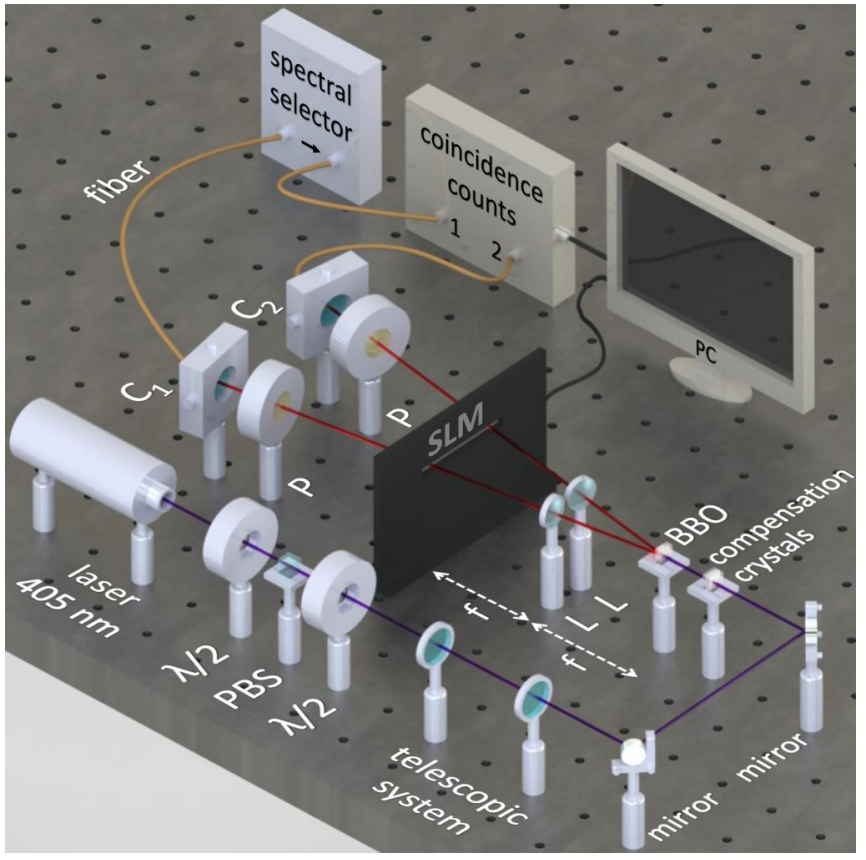

FIG. 2. Schematic diagram of the experimental setup. A 405 $\mathrm{nm} \mathrm{cw}$ laser diode generates a pump beam which passes through a half-wave plate $(\lambda / 2)$, a polarizing beam-splitter cube (PBS), and another half-wave plate. Then it is collimated by a telescopic system composed of two lenses $L_{t 0}$ and $L_{t 1}$. The beam passes through a series of compensation crystals and then it interacts with two 1-mm-long BBO crystals generating photons centered at $810 \mathrm{~nm}$ via PDC. Each branch passes through a lense $L$ with focal length $f$, a spatial light modulator (SLM), and a polarizer (P). Photons are finally focused into two multimode fibers through the couplers $C_{1}$ and $C_{2}$ : the first is directly linked to a homemade single-photon counting module, the second is sent to a spectral selector and then to the counting module.

in Eq. (5). Then, by taking the partial trace over the spatial degrees of freedom, we obtain

$$
\begin{aligned}
\rho_{\mathrm{S}}(t)= & \frac{1}{2}(|\mathrm{HH}\rangle\langle\mathrm{HH}|+| \mathrm{VV}\rangle\langle\mathrm{VV}| \\
& \left.+p \Gamma(t)|\mathrm{HH}\rangle\left\langle\mathrm{VV}\left|+p \Gamma^{*}(t)\right| \mathrm{VV}\right\rangle\langle\mathrm{HH}|\right),
\end{aligned}
$$

where $p$ is a parameter quantifying the entanglement in the initial state,

$$
\rho_{\mathrm{S}}(0)=p \rho_{0}+(1-p) \rho_{\text {mix }},
$$

where $\rho_{\text {mix }}=\frac{1}{2}(|\mathrm{HH}\rangle\langle\mathrm{HH}|+| \mathrm{VV}\rangle\langle\mathrm{VV}|)$. In our case $p$ is close to 1 and the procedure we use to purify the state is described in Refs. [24,25]. The decoherence function $\Gamma(t)$ depends on the spatial correlations between the two photons and on the stochastic realizations:

$$
\Gamma(t)=\sum_{j k}\left|f_{j k}\right|^{2} e^{i\left[\varphi_{1}\left(x_{1 j}, t\right)+\varphi_{2}\left(x_{2 k}, t\right)\right]},
$$

where the distribution

$$
\begin{aligned}
\left|f_{j k}\right|^{2}= & N_{0} \exp \left\{-\frac{2\left[\left(j-j_{0}\right)-\left(k-k_{0}\right)\right]^{n}}{w_{\mathrm{cp}}^{n}}\right\} \\
& \times \exp \left\{-\frac{2\left(j-j_{0}\right)^{2}}{w_{p}^{2}}-\frac{2\left(k-k_{0}\right)^{2}}{w_{p}^{2}}\right\}
\end{aligned}
$$

takes into account the size of the coupled PDC $w_{p}$ and spatial correlation between the photons (i.e., the number of correlated pixels) $w_{\mathrm{cp}}$ (see Appendixes $\mathrm{A}$ and $\mathrm{B}$ for details and the derivation). The first factor is a super-Gaussian of order $n$, while $j_{0}$ and $k_{0}$ are the central pixels on the SLM for each PDC branch. Finally, $N_{0}$ is a normalization factor to assure that $\sum_{j k}\left|f_{j k}\right|^{2}=1$. It is now clear that we may simulate the LtG transition using two different strategies, either by controlling the realizations of the noise on the two paths of the PDC, or by tuning the number of correlated pixels.

\section{RESULTS}

Let us start by explaining the role of the SLM in encoding the stochastic process into the pixels. We figuratively divide the SLM in two parts, both made of 320 pixels. The first set is dedicated to the first qubit and the pixels are indexed by an integer $j$ that goes from 0 to 319. The second part is dedicated to the second qubit and the pixels are labeled by $k$ that goes from 320 to 639 . Call $d$ the width of the pixel; the two positions $x_{1}$ and $x_{2}$ are $x_{1 j}=d j, x_{2 k}=d(640-k)$. This allows us to directly consider the symmetry of the spatial correlations between the photons in the notation.

The first step in order to send the same noise on the correlated pixels in the two parts of the mask is to experimentally find out the central pixels $j_{0}$ and $k_{0}$. The central pixels are the reference for the definition of the phases $\varphi_{1}$ and $\varphi_{2}$. In particular we set

$$
\varphi_{1}\left(x_{1, j_{0}+\Delta}, t\right)=\varphi_{2}\left(x_{2, k_{0}+\Delta}, t\right)=\varphi(\Delta, t),
$$

where $\Delta$ is an integer shift with respect to the references and $\varphi$ is a phase function defined over 320 points. The first method we use to simulate the $\mathrm{LtG}$ transition consists of introducing an integer shift $\delta$ on the array of phases $\varphi_{2}$ imprinted on the second side of the SLM. Setting $\delta=0$ the correlated pixels see the same noise and we mimic the case where the environments are fully correlated. When $\delta$ is increased, the two environments become progressively less correlated. It follows that, as the value of $\delta$ is decreased from a large value to zero, we obtain the LtG transition. The function $\Gamma$ is now a function of $\delta$ and we have

$$
\Gamma(\delta, t)=\sum_{j k}\left|f_{j k}\right|^{2} e^{i\left[\varphi_{1}\left(x_{1 j}, t\right)+\varphi_{2}\left(x_{2 k+\delta}, t\right)\right]} .
$$

Since this technique is effective for spatial correlation lengths $w_{\mathrm{cp}}$ smaller or equal with respect to the typical spatial variation of the function $\varphi$, we use a spectral width of $\Delta \lambda=$ $15 \mathrm{~nm}$, resulting in a $w_{\mathrm{cp}}$ of about three pixels and use a function $\varphi$ that changes value every three pixels. By reducing the spectrum width it is possible to obtain smaller values of $w_{\mathrm{cp}}$ at the price of reducing counts and, in turn, increasing fluctuations.

At first, let us consider the experimental realization of the local-to-global transition in the case of the RTN with $\gamma=0$ using the $\delta$-shift technique. Upon imposing $\delta=0$ we obtain two fully correlated environments, and using Eq. (4) we have

$$
\left|\Gamma_{\mathrm{GE}}(t)\right|_{\gamma=0}=\left|\left\langle e^{4 i \varphi(t)}\right\rangle\right|=|\cos (4 t)| .
$$

Indeed, due to the RTN noise, the only two possible values for $\varphi$ are $t$ and $-t$. The blue and the red curves in Fig. 3(b) are 


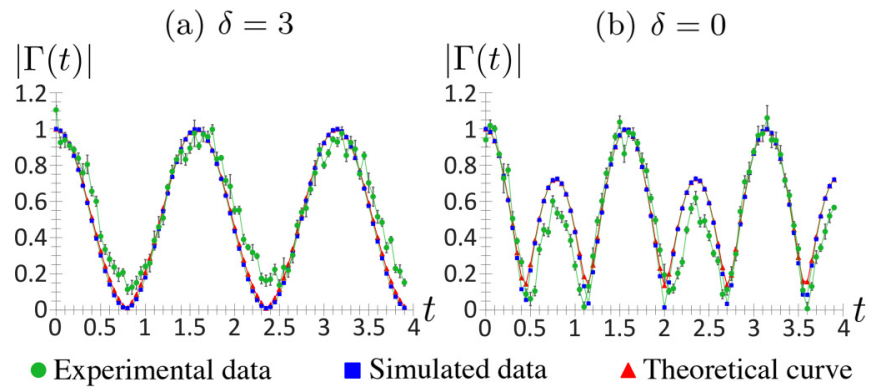

FIG. 3. Local-to-global noise transition by translation of the realizations $\varphi(t)$ for the RTN with $\gamma=0$ and $\Delta \lambda=15 \mathrm{~nm}$. The time $t$ is in arbitrary units. The green dots represent the experimental data, while the blue squares are the corresponding simulations for $|\operatorname{Re}[\Gamma(t)]|$. The red triangles are $|\Gamma(t)|$. In the left panel we show results for $\delta=3$, i.e., the two environments are not correlated. The right panel is $\delta=0$, i.e., fully correlated environments.

respectively $|\Gamma|$ and $|\operatorname{Re}\{\Gamma\}|$, obtained theoretically by using the experimental values for $w_{\mathrm{cp}}$ and $w_{p}$ of 3 and 20 pixels, respectively. The comparison between the red and the blue curves shows that the $\Gamma$ function is real, as it would be in the ideal case with an infinite number of realizations. To obtain this result it is necessary to select the $\varphi$ function with a balanced number of positive and negative realization at time $t=0$. When $\delta=3$ [Fig. 3(a)] the environments are not correlated. In this case the two qubits see two different phases (indeed the shift $\delta$ makes the two quantities $\varphi_{1}$ and $\varphi_{2}$ completely different at the correlated positions). Using Eq. (4) we have

$$
\left|\Gamma_{\mathrm{LE}}(t)\right|_{\gamma=0}=\left\langle e^{2 i \varphi(t)}\right\rangle^{2}=\cos (2 t)^{2} .
$$

Notice that the second peak in Fig. 3(b) does not reach the value 1 due to the under-sampling of the noise realizations [26].

The second strategy to obtain the LtG transition consists in increasing the number of correlated pixels $w_{\mathrm{cp}}$ while fixing $\delta=0$ and the number of repeated pixels in the function $\varphi$. To increase $w_{\text {cp }}$ we increase the width of the PDC spectrum by acting on the spectral selector, see Appendix $\mathrm{C}$. When the spectral width is $\Delta \lambda=15 \mathrm{~nm}$, the number of correlated pixels is equal to the number of repeated pixel in the $\varphi$ function and the two qubits see the same environment. By progressively increasing the value of $\Delta \lambda, w_{\mathrm{cp}}$ becomes larger than the number of repeated pixels in $\varphi$ and the two qubits see different environments. Indeed, on the two photons are imprinted different phases.

In Fig. 4, we show the experimental realization of the transition using both techniques. In the left column the $\delta$ shift method is used, and in the right column the transition is obtained changing the PDC spectral width. We note that, moving from local noise to global noise, the rising of the new peaks is evident. Here $\gamma=0.12$ in order to show a case with a nonstationary RTN noise. Fluctuations in the experimental data are mostly due to the strong dependence on the central pixel. Indeed, the center of the PDC beam may be shifted from the center of the pixel itself for a fraction of the pixel's length: this has been taken into account in the simulations but
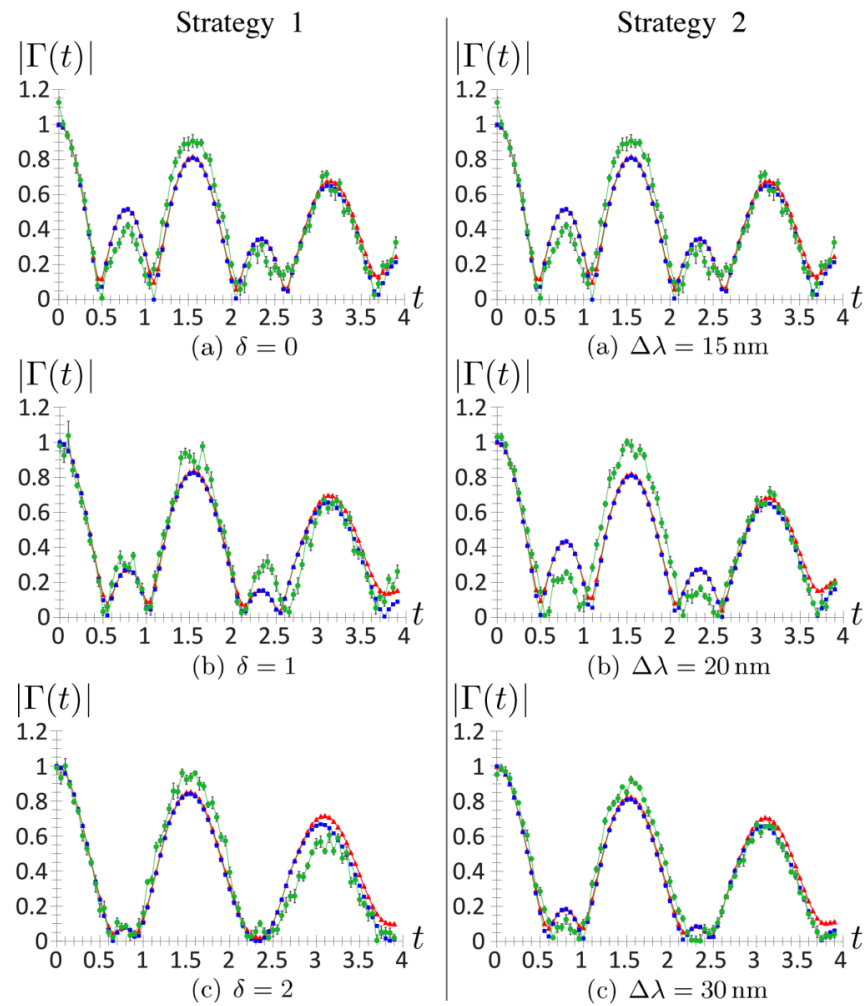

1.2

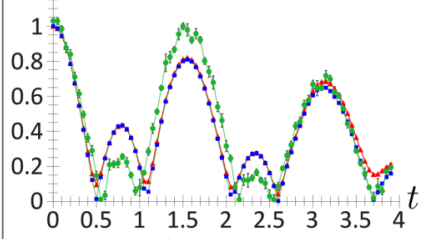

(b) $\Delta \lambda=20 \mathrm{~nm}$

$|\Gamma(t)|$

1.2

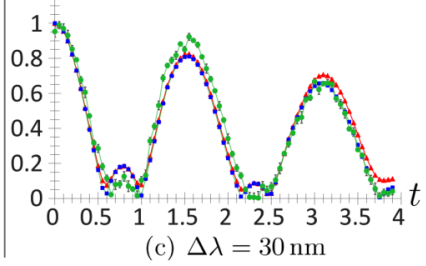

- Experimental data

- Simulated data

$\Delta$ Theoretical curve

FIG. 4. Experimental implementation of the transition from global to local noise in the case of RTN with $\gamma=0.12$. The time $t$ is in arbitrary units. In the left column the $\delta$ shift-strategy is used. On the right column the transition is obtained by changing the spectral width $\Delta \lambda$.

it changes from one measure to another and it is not possible to estimate it with sufficient precision.

\section{CONCLUSIONS}

We have experimentally demonstrated the transition from local-to-global decoherence in an all-optical two-qubit quantum simulator subject to classical noise. We exploited the spatial degrees of freedom of the PDC photons to implement the noise realizations while the photon polarizations encoded the two qubits. In particular, thanks to the high control of the PDC width and of the spatial correlations among pixels of the SLM, we have been able to implement two different strategies for the noise transition, either involving the building of correlations between environments or the tuning of the PDC spectral width. Besides RTN, which we used as a test-bed for our simulator, any kind of classical noise may be implemented, making our scheme suitable to simulate a wide range of dynamics involving super- and semiconducting qubits that are of the utmost importance for quantum technologies.

Our results also pave the way for the realization of manyqubit simulators and open up the chance of exploring the dynamics of multipartite entanglement as well as studying the robustness of quantum features against decoherence. In multiqubit systems the LtG transition takes a broader meaning with subgroups of qubits that may feel the same noise while 


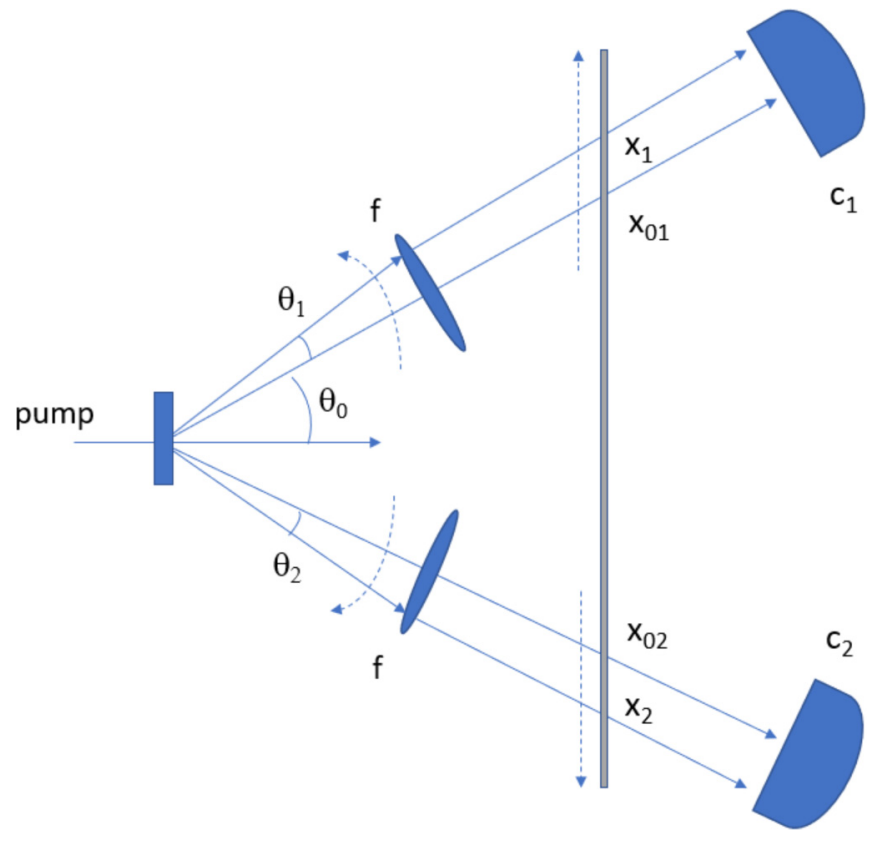

FIG. 5. The geometry of the parametric-down-conversion scheme.

others are subject local fluctuations. In this case spatial correlations becomes the key element that governs the dynamics [27]. More generally, the ability to monitor and control the LtG transition is a fundamental step in understanding decoherence, especially in the context of reservoir engineering, where the noise is tailored or to improve performances of specific protocols [28-30].

\section{ACKNOWLEDGMENTS}

This work has been partially supported by UniMI through Project No. PSR2017-DIP-008, and by MAECI through Project No. PGR06314-ENYGMA. The authors thank Dario Tamascelli for discussions.

\section{APPENDIX A: TWO-PHOTON SPATIAL CORRELATIONS}

Figure 5 shows the geometrical configuration of the two photons generated by PDC. The two angles $\theta_{1}$ and $\theta_{2}$ are the angular shifts with respect to the PDC central angle $\theta_{0}$ defined by the phase-matching condition. The coordinates $x_{1}, x_{2}$ and $x_{01}, x_{02}$ are respectively the positions and the references on the SLM plane. The arrows represent the orientations we use for the axes. The two-photon state can be written as [23]

$$
\begin{aligned}
|\psi\rangle= & \frac{1}{\sqrt{2}} \int d \omega d \theta_{1} d \theta_{2} \tilde{A}\left(\Delta k_{\perp}\right) \operatorname{Sinc}\left(\Delta k_{\|} L / 2\right) \\
& \times\left[\left|H, \theta_{1}, \omega\right\rangle\left|H, \theta_{2},-\omega\right\rangle\right. \\
& \left.+e^{i \Phi\left(\theta_{1}, \theta_{2}\right)}\left|V, \theta_{1}, \omega\right\rangle\left|V, \theta_{2},-\omega\right\rangle\right]
\end{aligned}
$$

where, up to first order in frequency and angle,

$$
\begin{gathered}
\Delta k_{\|}=-\omega_{p}^{0} \theta^{0}\left(\theta_{1}+\theta_{2}\right) /(2 c), \\
\Delta k_{\perp}=\omega_{p}^{0}\left(\theta_{1}-\theta_{2}\right) /(2 c)+2 \theta^{0} \omega / c .
\end{gathered}
$$

$\omega_{p}^{0}$ is the pump frequency $(405 \mathrm{~nm})$ and $\omega$ is the frequency shift with respect the PDC central frequency $\omega_{p} / 2(810 \mathrm{~nm})$, $c$ is the speed of light. and the phase term $\Phi\left(\theta_{1}, \theta_{2}\right)$ is due to the different optical paths followed by the pairs of photons generated in the first and second crystal.

The function $\tilde{A}$ comes from the integration along the transverse coordinate [31] and it is the Fourier transform of the pump spatial amplitude, the Sinc function comes from the integration along the longitudinal coordinate inside the crystal ( $L$ is the crystal length), and $\Delta k_{\|}$and $\Delta k_{\perp}$ are the shifts with respect to the phase-matching condition of the longitudinal and the transverse momentum of the two photons. Finally, we obtain $\Phi=0$ by using the purification method explained in Refs. [24,25]. Due to the pump spot dimension $\left(w_{\text {pump }} \approx\right.$ $0.6 \mathrm{~mm})$ and the crystal length $(1 \mathrm{~mm})$ employed in our experimental configuration, the angular correlations depend mainly on the function $\tilde{A}$ and in turn by $\Delta k_{\perp}$. Considering that $\Delta k_{\perp}$ is a function of $\omega$, it is simple to get that, by enlarging the PDC spectrum width, we progressively lose the angular correlations. Moreover, when the spectrum width goes to zero, the angular correlations depend directly on the width of the pump spot via its Fourier transform $\tilde{A}$. To obtain both the PDC width $w_{p}$ and the spatial correlations, we define: $F\left(\theta_{1}, \theta_{2}\right)=\int d \omega\left|\tilde{A}\left(\Delta k_{\perp}\right) \operatorname{Sinc}\left(\Delta k_{\|} L / 2\right)\right|^{2}$. Before proceeding with the calculation, we have to switch from angular to spatial coordinates. Due to the fact that the lens $f$ is placed at distance $f$ both from the crystals and the SLM, we have $\Delta x_{1}=x_{1}-x_{01}=f \theta_{1}$ and $\Delta x_{2}=x_{2}-x_{02}=f \theta_{2}$ and we can write $F$ as a function of $\Delta x_{1}$ and $\Delta x_{2}$.

To estimate the PDC width $w_{p}$ we define the function: $F_{p}\left(\Delta x_{1}\right)=\int d \Delta x_{2} F\left(\Delta x_{1}, \Delta x_{2}\right)$. This function is the same for the two paths of the PDC and is well approximated by a Gaussian profile. It gives the probability to detect a photon vs the spatial coordinates, so its width is the PDC width. From a numerical approach we obtain $w_{p} \approx 20$ pixels. This number is confirmed by a direct measure of the PDC profile. We note also that this width is directly connected with the Sinc function and is only weakly dependent on the collection spatial efficiency. This is a consequence of the fact that, in our experimental scheme, the PDC cone is forced to remain in a little area due to the presence of the lens $f$.

Now we can face the derivation of the correlation length $w_{\text {cp }}$. This quantity is of fundamental importance in our work and it gives the probability to detect a photon within a definite interval when the other photon is found in a definite position (a pixel in our case). In particular, it is clear that in order to define a proper $\varphi$ function, we need an experimental apparatus able to generate a correlation length of only few pixels, and for this reason we use the configuration with the lenses $f$ between the crystals and the SLM. The correlation length $w_{\mathrm{cp}}$ has two contributions, one connected directly to the function $F$ and the other one connected to the pump dimension. The first contribution is the width $\tilde{w}_{\mathrm{pc}}$ of the function $F_{c p}=F\left(\Delta x_{1}, 0\right)$. About this function it is important to say that this width does not change if we integrate position 2 along the dimension of one pixel. $\tilde{w}_{\text {cp }}$ increases with the spectrum width and the profile of $F_{c p}$ is well reproduced by a Gaussian when the spectrum width is less than $15 \mathrm{~nm}$ and it is well reproduced by a super-Gaussian with $n=4$ for bigger spectrum width. This result depends on the fact that, with our spectrum selector, we 
obtain a quasirectangular profile of the PDC spectrum. The second contribution is related to the pump-spot dimension. About this we have to consider that the PDC is generated not only in one point in the transversal direction but along the pump profile [32]. The point is that the spatial coherence properties of the pump are directly transferred into the PDC. In a naive picture we can say that the single mode of the pump is transferred into the single mode (defined by the direction $\theta$ ) of the PDC. This means that the lens $f$ focuses this single mode on the SLM plane with a dimension $w_{c p}^{0}=\frac{\lambda_{0} f}{\pi w_{\text {pump }}} \approx 1$ pixel where $\lambda_{0}=810 \mathrm{~nm}$. Without focusing, $w_{\mathrm{cp}}^{0}$ would be equal to the pump dimension; indeed, in our case we have a well-collimated pump. An alternative scheme would be to use a focused pump but we note that in this case we have to put the lens before the crystals, obtaining in turn a bigger dimension on the SLM plane. Finally, considering these two contributions, we have

$$
w_{\mathrm{cp}}=\sqrt{\left(\tilde{w}_{\mathrm{cp}}\right)^{2}+\left(w_{\mathrm{cp}}^{0}\right)^{2}},
$$

so we can write

$$
F\left(\Delta x_{1}, \Delta x_{2}\right)=e^{-\frac{2\left(\Delta x_{1}-\Delta x_{2}\right)^{n}}{w_{\mathrm{cp}}^{n}}} e^{-\frac{2\left(\Delta x_{1}\right)^{2}}{w_{p}^{2}}} e^{-\frac{2\left(\Delta x_{2}\right)^{2}}{w_{p}^{2}}}
$$

(without considering a normalization factor). To obtain Eq. (5) of the main text, we have only to demonstrate that $\left|f_{j k}\right|^{2}=$ $F\left(\Delta x_{1}, \Delta x_{2}\right)$, and we can easily see that this equality is assured by the fact that the phase $\Phi$ is not a function of $\omega$.

\section{APPENDIX B: MEASURE OF THE COHERENCE FACTOR}

If the system is in the state $\rho_{\mathrm{S}}(t)$, and the polarizers are both at $45^{\circ}$, the detection probability (considering the quantum efficiency $\mathrm{QE}=1$ ) is

$$
p_{++}=\frac{1}{4}(1+p \operatorname{Re}\{\Gamma\}),
$$

while, if one polarizer is at $45^{\circ}$ and the other at $135^{\circ}$, it is

$$
p_{+-}=\frac{1}{4}(1-p \operatorname{Re}\{\Gamma\}) .
$$

Then, the coincidence counts for second in the two cases are

$$
\begin{aligned}
& N_{++}=N_{0}[1+p(\operatorname{Re}\{\Gamma\})], \\
& N_{+-}=N_{0}[1-p(\operatorname{Re}\{\Gamma\})],
\end{aligned}
$$

where $N_{0}$ is obtained directly from the experimental counts and it takes into account the spatial-spectral quantum efficiencies of the detection system. So we can infer information about $\Gamma$ from the visibility:

$$
V=\left|\frac{N_{++}-N_{+-}}{N_{++}+N_{+-}}\right|=p|\operatorname{Re}\{\Gamma\}| .
$$

In the ideal case (without the under-sampling effect [26]), $\Gamma$ is a real quantity. This case can be experimentally recovered by taking the array of noise phases $\varphi$ with zero mean. In the graphs in Figs. 3 and 4 (in the main text) we show the comparison between the theoretical curves of $|\operatorname{Re}\{\Gamma\}|$ and $|\Gamma|$ to put in evidence the effectiveness of this method.

\section{APPENDIX C: MEASURE OF THE NUMBER OF CORRELATED PIXELS}

Let us introduce in the first half of the SLM a rectangular function which switches $\pm \pi / 4$ every $n_{r}=5$ pixels, and in the second half the same function shifted by $h$. Therefore $\Gamma$ is a function of $h$. By the measurements of $N_{++}=N_{++}(h)$ and
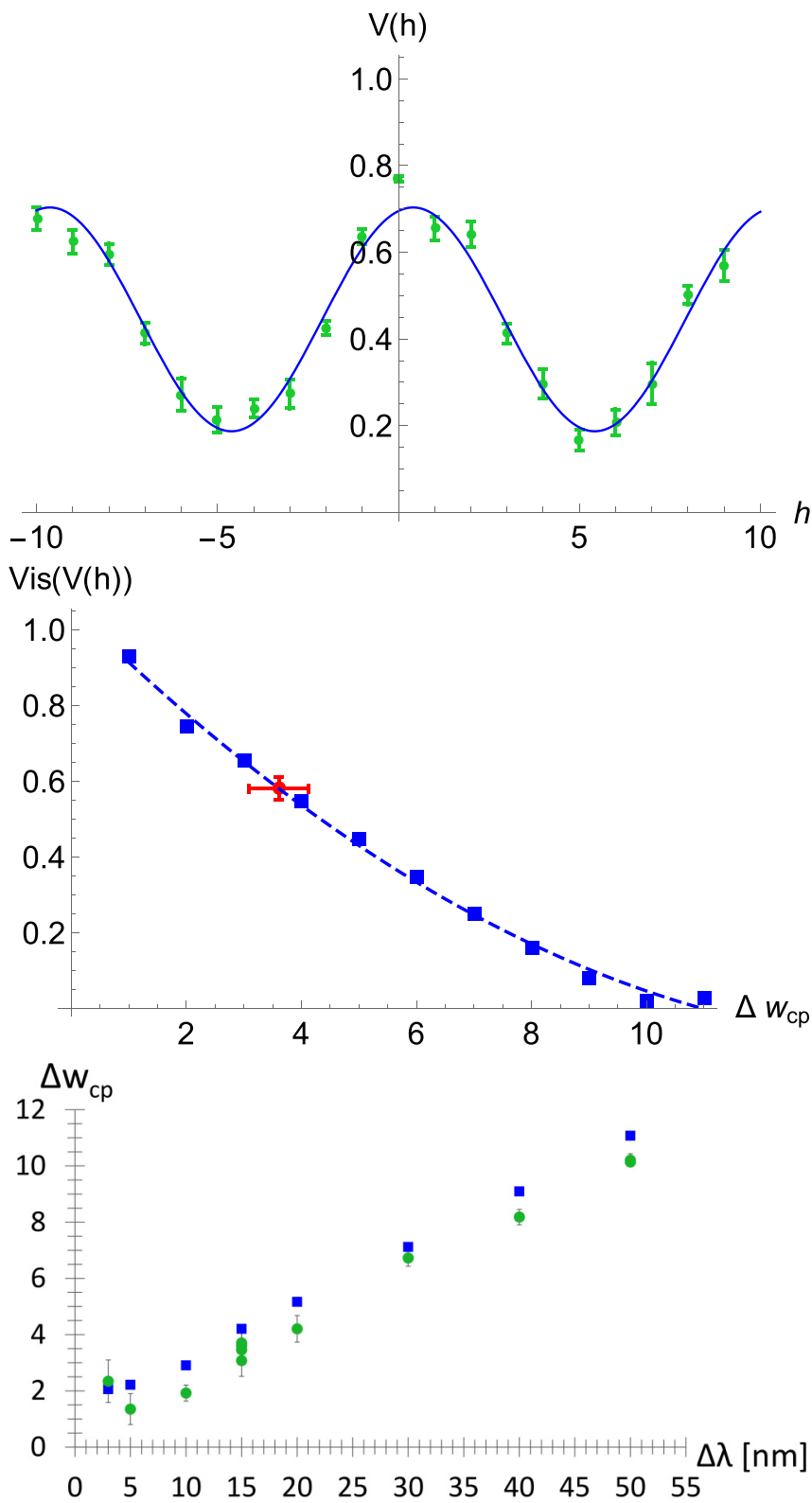

FIG. 6. (top panel) The green dots represent the measured values of $V(h)$ for $\Delta \lambda=15 \mathrm{~nm}$ and $p=0.927$, while the blue line is the fitting sine wave. In the present case, it gives $\operatorname{Vis}(V(h))=$ $0.58 \pm 0.03$. (central panel) The blue squares represent the value of $\operatorname{Vis}(V(h))$ obtained by simulation for $\Delta \lambda=15 \mathrm{~nm}$ and $q=0.927$, while the blue dashed line is the fitting polynomial curve. The red dot is the measured value of visibility. In the present case, the extrapolated number of correlated pixel is $w_{\mathrm{cp}}=3.1 \pm 0.5$. (bottom panel) Number of correlated pixels as a function of the spectral width of the PDC: the green dots are the experimental data, the blue squares are the simulated data. 
$N_{+-}=N_{+-}(h)$ for $h=-10,-9, \ldots, 9$, where each point is an average of four measures and each measure has an acquisition time of $8 \mathrm{~s}$, we calculate $V=V(h)$, as shown in the left panel of Fig. 6. The visibility of $V(h)$ is a decreasing function of the number of correlated pixels $w_{\mathrm{cp}}$ and can be simulated as shown in the central panel of Fig. 6, so that from the experimental value of $\operatorname{Vis}(V(h))$ one can extrapolate $w_{\text {cp. }}$. Repeating the experiment for different apertures of the spectral selector, we obtain the number of correlated pixels as a function of $\Delta \lambda$ (see the right panel of Fig. 6). The experimental data undergo a saturation for small $\Delta \lambda$ because of the effect of the transversal width of the pump.
[1] I. Buluta and F. Nori, Science 326, 108 (2009).

[2] I. M. Georgescu, S. Ashhab, and F. Nori, Rev. Mod. Phys. 86, 153 (2014).

[3] D. Jaksch and P. Zoller, Ann. Phys. (NY) 315, 52 (2005).

[4] I. Kassal, J. D. Whitfield, A. Perdomo-Ortiz, M.-H. Yung, and A. Aspuru-Guzik, Annu. Rev. Phys. Chem. 62, 185 (2011).

[5] A. Aspuru-Guzik and P. Walther, Nat. Phys. 8, 285 (2012).

[6] R. Blatt and C. F. Roos, Nat. Phys. 8, 277 (2012).

[7] A. A. Houck, H. E. Türeci, and J. Koch, Nat. Phys. 8, 292 (2012).

[8] D. Crow and R. Joynt, Phys. Rev. A 89, 042123 (2014).

[9] S. Cialdi, M. A. C. Rossi, C. Benedetti, B. Vacchini, D. Tamascelli, S. Olivares, and M. G. A. Paris, Appl. Phys. Lett. 110, 081107 (2017).

[10] Z.-D. Liu, H. Lyyra, Y.-N. Sun, B.-H. Liu, C.-F. Li, G.-C. Guo, S. Maniscalco, and J. Piilo, Nat. Commun. 9, 3453 (2018).

[11] T. Yu and J. H. Eberly, Phys. Rev. B 68, 165322 (2003).

[12] C. Benedetti, F. Buscemi, P. Bordone, and M. G. A. Paris, Int. J. Quantum Inform. 10, 1241005 (2012).

[13] C. Benedetti, F. Buscemi, P. Bordone, and M. G. A. Paris, Phys. Rev. A 87, 052328 (2013).

[14] A. C. S. Costa, M. W. Beims, and W. T. Strunz, Phys. Rev. A 93, 052316 (2016).

[15] S. Daniotti, C. Benedetti, and M. G. A. Paris, Eur. Phys. J. D 72, 208 (2018).

[16] D. P. S. McCutcheon, A. Nazir, S. Bose, and A. J. Fisher, Phys. Rev. A 80, 022337 (2009).

[17] A. Streltsov, H. Kampermann, and D. Bruss, Phys. Rev. Lett. 107, 170502 (2011).
[18] R. Lo Franco, B. Bellomo, S. Maniscalco, and G. Compagno, Int. J. Mod. Phys. B 27, 1345053 (2013).

[19] C. Addis, P. Haikka, S. McEndoo, C. Macchiavello, and S. Maniscalco, Phys. Rev. A 87, 052109 (2013).

[20] M. A. C. Rossi, C. Benedetti, and M. G. A. Paris, Int. J. Quantum Inform. 12, 1560003 (2014).

[21] F. Galve, A. Mandarino, M. G. A. Paris, C. Benedetti, and R. Zambrini, Sci. Rep. 7, 42050 (2017).

[22] P. G. Kwiat, E. Waks, A. G. White, I. Appelbaum, and P. H. Eberhard, Phys. Rev. A 60, R773 (1999).

[23] A. Smirne, S. Cialdi, G. Anelli, M. G. A. Paris, and B. Vacchini, Phys. Rev. A 88, 012108 (2013).

[24] S. Cialdi, D. Brivio, and M. G. A. Paris, Appl. Phys. Lett. 97, 041108 (2010).

[25] S. Cialdi, D. Brivio, and M. G. A. Paris, Phys. Rev. A 81, 042322 (2010).

[26] M. A. C. Rossi, C. Benedetti, S. Cialdi, D. Tamascelli, S. Olivares, B. Vacchini, and M. G. A. Paris, Int. J. Quantum Inform. 15, 1740009 (2017).

[27] M. A. C. Rossi, C. Benedetti, M. Borrelli, S. Maniscalco, and M. G. A. Paris, Phys. Rev. A 96, 040301(R) (2017).

[28] J. F. Poyatos, J. I. Cirac, and P. Zoller, Phys. Rev. Lett. 77, 4728 (1996).

[29] D. de Falco and D. Tamascelli, J. Phys. A: Math. Theor. 46, 225301 (2013).

[30] S. McEndoo, P. Haikka, G. de Chiara, M. Palma, and S. Maniscalco, Europhys. Lett. 101, 60005 (2013).

[31] S. Cialdi, D. Brivio, A. Tabacchini, A. M. Kadhim, and M. G. A. Paris, Opt. Lett. 37, 3951 (2012).

[32] A. K. Jha and R. W. Boyd, Phys. Rev. A 81, 013828 (2010). 